The Astrophysical Journal, 640:956-965, 2006 April 1

(C) 2006. The American Astronomical Society. All rights reserved. Printed in U.S.A.

\title{
ENSEMBLE CHARACTERISTICS OF THE ZZ CETI STARS
}

\author{
Anjum S. Mukadam ${ }^{1}$ \\ Department of Astronomy, University of Washington, Seattle, WA 98195-1580; anjum@astro.washington.edu \\ M. H. Montgomery and D. E. Winget \\ Department of Astronomy, University of Texas at Austin, Austin, TX 78712 \\ S. O. KEPLER \\ Instituto de Física, Universidade Federal do Rio Grande do Sul, 91501-970 Porto Alegre RS, Brazil \\ AND \\ J. C. Clemens \\ Department of Physics and Astronomy, University of North Carolina, Chapel Hill, NC 27599 \\ Received 2005 July 18; accepted 2005 December 5
}

\begin{abstract}
We present the observed pulsation spectra of all known noninteracting ZZ Ceti stars (hydrogen atmosphere white dwarf variables [DAVs]) and examine changes in their pulsation properties across the instability strip. We confirm the well-established trend of increasing pulsation period with decreasing effective temperature across the ZZ Ceti instability strip. We do not find a dramatic order-of-magnitude increase in the number of observed independent modes in ZZ Ceti stars, traversing from the hot to the cool edge of the instability strip; we find that the cool DAVs have one more mode on average than the hot DAVs. We confirm the initial increase in pulsation amplitude at the blue edge and find strong evidence of a decline in amplitude prior to the red edge. We present the first observational evidence that ZZ Ceti stars lose pulsation energy just before pulsations shut down at the empirical red edge of the instability strip.
\end{abstract}

Subject headings: stars: oscillations — stars: variables: other — white dwarfs

\section{INTRODUCTION}

Asteroseismology is the only systematic technique available for studying the insides of a star. Pulsators are fortunately found all over the H-R diagram and allow us the opportunity to look inside different stars in various evolutionary stages. White dwarf stars are the stellar remains of $98 \%-99 \%$ of stars in the sky (Weidemann 1990) and contain an archival record of their mainsequence lifetime. Pulsating white dwarf stars serve as effective instruments to harness this archival record.

White dwarf spectra reveal that $80 \%$ of them have atmospheres dominated by hydrogen (DA's; Fleming et al. 1986). Hydrogen atmosphere DA white dwarfs are observed to pulsate in an instability strip located in the temperature range 10,800 $12,500 \mathrm{~K}$ for $\log g \approx 8$ (Bergeron et al. 1995, 2004; Koester \& Allard 2000; Koester \& Holberg 2001; Mukadam et al. 2004a). Recent studies have determined that the DA instability strip is only about $1000-1200 \mathrm{~K}$ in width (Mukadam et al. 2004a; Gianninas et al. 2005). These DA variables (DAVs) are known as the ZZ Ceti stars, after the prototype of the class, ZZ Ceti (Ross 548).

In this paper we gather the pulsation spectra of all known noninteracting ZZ Ceti stars with the purpose of studying their ensemble pulsation characteristics and how these properties change across the instability strip. Such a systematic study of the ZZ Ceti pulsators was first undertaken by pioneers in the field such as Robinson (1980), McGraw et al. (1981), and Winget \& Fontaine (1982). Clemens (1993) was the first to systematically demonstrate the distinct behavior of pulsation periods and amplitudes as a function of temperature for a significant sample of

\footnotetext{
${ }^{1}$ Hubble Fellow.
}

DAVs. The sample size of known DAVs is now 3 times larger than the sample used in the last such characterization of the ZZ Ceti pulsators by Kanaan et al. (2002).

We now also have better and internally consistent temperatures for these pulsators, calculated using the ML $2 / \alpha=0.6$ convection description (see Bergeron et al. 2004; Kleinman et al. 2004). This prescription for convection gives the best internal consistency between optical and ultraviolet (UV) effective temperatures, trigonometric parallaxes, $V$ magnitudes, and gravitational redshifts (Bergeron et al. 1995; Koester \& Vauclair 1997; Bergeron \& Lamontagne 2003; Fontaine et al. 2003). These two reasons compel us to reexamine the pulsation characteristics of the instability strip.

\subsection{Defining Our ZZ Ceti Samples}

Of the 35 new ZZ Ceti variables published in Mukadam et al. (2004b), HS 0951+1312, HS 0952+1816, WD 1443+0134, WD 1524-0030, and WD 2350-0054 do not have reliable spectroscopic $T_{\text {eff }}$ and $\log g$ fits (see Mukadam et al. 2004b, 2004a). Our sample of new ZZ Ceti variables discovered using spectra from the Sloan Digital Sky Survey (SDSS) then consists of 30 DAVs from Mukadam et al. (2004b) and 11 DAVs from Mullally et al. (2005). For these 41 DAVs, D. Eisenstein derived a homogeneous and internally consistent set of $T_{\text {eff }}$ and $\log g$ values from the SDSS using model atmospheres from D. Koester (see Kleinman et al. 2004). We carefully and consistently reanalyzed all of our time-series photometry data on these pulsators, acquired using the prime-focus CCD camera Argos (Nather \& Mukadam 2004) on the $2.1 \mathrm{~m}$ telescope at McDonald Observatory. We hereafter refer to this set of $41 \mathrm{DAVs}$ as the SDSS ZZ Ceti sample with homogeneous spectroscopic fits and homogeneous time-series photometry. 
Bergeron et al. (2004) and Gianninas et al. (2005) have published internally consistent temperatures and $\log g$ fits for 39 DAVs using their latest model atmospheres; we hereafter refer to this second set of 39 DAVs as the BG04 ZZ Ceti sample. We compiled a corresponding set of 39 pulsation spectra from the literature and via private communication with our colleagues. The seismic data of the BG04 ZZ Ceti sample were acquired by different observers using different instruments and telescopes. However, a substantial amount of time-series data exists on most DAVs in this sample, and we utilized practically all published pulsation spectra to carefully derive well-averaged values of weighted mean period and pulsation amplitudes, which we present in this paper.

Mukadam et al. (2004a) show evidence of a relative shift of about $200 \mathrm{~K}$ between the SDSS and BG04 ZZ Ceti instability strips, which also differ in shape and width. The spectra of ZZ Ceti stars in these samples were analyzed using different techniques and with different model atmospheres. As homogeneity is imperative, we cannot merge these two samples for analyses that involve the spectroscopic temperature. However, we can merge these two samples when formulating plots based solely on seismic data, such as Figure 3.

During the writing of this manuscript, 25 new ZZ Ceti stars were submitted for publication in separate papers, Silvotti et al. (2005, 2006), Castanheira et al. (2005), Kepler et al. (2005), and Gianninas et al. (2005). This brings the total number of known noninteracting ZZ Ceti stars to 107 . Twenty of the new DAVs in these papers come from the SDSS. Their spectroscopic $T_{\text {eff }}$ and $\log g$ values were derived using the same technique as was used on the 41 SDSS DAVs discovered previously, but with a different algorithm (version auto23 vs. auto21, used earlier). Hence, we cannot include the new DAVs in our SDSS ZZ Ceti sample for plots based on effective temperature, such as Figures 1 and 2. Also, we find that the main purpose of the pulsation spectra presented in these papers is to demonstrate discovery of variability. Such pulsation spectra may be incomplete and are not well suited for direct inclusion in most plots of this paper, where ensemble homogeneity plays a big role. ${ }^{2}$ However we include these variables in Figure 3 (based on seismic data) for completeness, and show the net effect of the inclusion on the original plot.

\subsection{Methodology: Using Only Linearly Independent Pulsation Frequencies}

Brickhill (1992), Brassard et al. (1995), Wu (2001), and Montgomery (2005) show that the nonlinear pulse shapes observed in some variable white dwarfs may arise as a result of relatively thick convection zones. Many frequencies are evident in these stars because the nonlinearities appear as harmonics and linear combinations in our Fourier transforms. We are interested in studying the pulsation characteristics of self-excited real modes in the context of this paper, so here we disregard all harmonics and linear combination frequencies in the observed pulsation spectra.

When our program detects a linear combination or harmonic in an observed pulsation spectrum, we typically hand pick the lowest amplitude mode as the linear combination mode. However, if we find a mode involved in two or more linear combi-

\footnotetext{
2 We added three new DAVs HS 1039+4112 (Silvotti et al. 2005), G232-38 (Gianninas et al. 2005), and GD 133 (Silvotti et al. 2006) to the original BG04 sample of 36 stars; this does not significantly affect the homogeneity of the BG04 sample, as the new DAVs constitute less than $10 \%$ of the sample. However, adding 20 new SDSS DAVs to the SDSS ZZ Ceti sample of 41 stars would significantly affect the homogeneity of the sample.
}

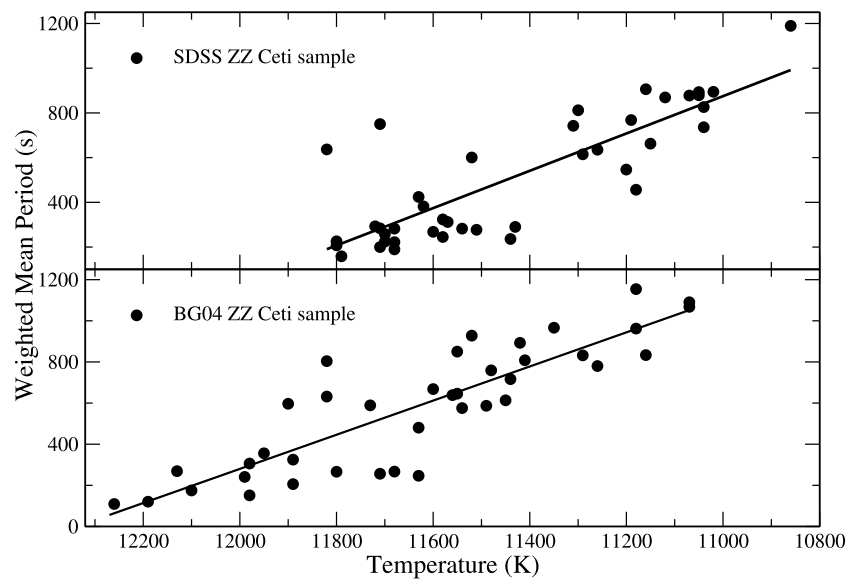

FIG. 1.-Weighted mean period of 41 newly discovered ZZ Ceti stars from the SDSS (top) and $39 \mathrm{ZZ}$ Ceti stars from the BG04 sample (bottom) vs. their spectroscopic temperature; each period was weighted by the observed amplitude. We note the distinct increase in mean pulsation period as DAVs cool across the ZZ Ceti instability strip.

nations, we consider it to be a linear combination mode even if it is not the lowest amplitude mode. Our simplistic approach could lead us to incorrect choices; we could be misinterpreting resonances as linear combination modes in a few cases. However, any small discrepancies in values evaluated for a single star should have a minimal impact on the conclusions we draw from the ensemble.

\section{CHANGES IN PULSATION PROPERTIES ACROSS THE ZZ CETI INSTABILITY STRIP}

Short pulsation periods $(100-300 \mathrm{~s})$ in the ZZ Ceti stars typically have pulsation amplitudes of a few percent or smaller. Long pulsation periods (600-1000 s) typically have large amplitudes, which can be as high as $10 \%$. This correlation between the pulsation periods and amplitudes of the ZZ Ceti stars has been established for a long time (Robinson 1979, 1980; McGraw 1980; Fontaine et al. 1982). However, the correlation of these properties with temperature had to wait more than a decade for sufficiently accurate determinations of ZZ Ceti temperatures. Subsequently, the distinct behavior of pulsation periods and amplitudes as a function of temperature was systematically demonstrated for a significant sample of DAVs by Clemens (1993) and more recently by Kanaan et al. (2002). We now demonstrate and discuss these trends for the new as well as previously known DAVs of our two homogeneous samples. We begin by showing how the number of observed independent modes changes across the instability strip.

\subsection{Number of Observed Independent Modes}

We expect a larger (than intrinsic) scatter in the number of independent modes we measure for an ensemble of ZZ Ceti stars due to varied detection efficiency. This partially arises because we use different instruments on different telescopes or observe for different durations of time and because the weather conditions and extinction values vary. A fraction of the scatter in our detection efficiency is caused by ZZ Ceti stars having different magnitudes and different pulsation amplitudes, and the fact that the pulsation amplitudes depend on the effective wavelength of observation (Robinson et al. 1982).

By using the SDSS ZZ Ceti sample alone, we reduce some of this expected scatter (see $\S 1.1$ ). To further reduce the scatter, 
we choose to limit ourselves to the magnitude range $17.8 \leq$ $g \leq 18.8$ and to a stellar mass $M_{\star}<1.0 M_{\odot}$ to exclude intrinsic low-amplitude DAVs (see $\S 2.3$ ). Reducing our magnitude range any more will take us further into the realm of small number statistics. For the subset of 23 SDSS DAVs that fall in the chosen range, we find an average of 2.5 modes per star for the hotter ZZ Ceti stars $(11,300-11,800 \mathrm{~K})$ and a slightly larger value of 3.1 modes per star for the cooler ZZ Ceti stars $(10,800$ $11,300 \mathrm{~K}$ ). If we consider all the SDSS DAVs in our sample, excluding the three massive variables (WD 0923+0120, WD 1711+6541, and WD 2159+1322), then we find an average of 2.9 modes per star for the hotter ZZ Ceti stars $(11,300-$ $11,800 \mathrm{~K}$ ) and 3.3 modes per star for the cooler ZZ Ceti stars $(10,800-11,300 \mathrm{~K})$, up to an amplitude limit of order a few millimodulation amplitudes (mma). This may seem contrary to published literature, but we are attempting to restrict ourselves to independent self-excited modes only, rather than plot the total number of observed periodicities in the star.

The period spacing for nonradial $g$-modes with $k \leq 3$ is larger than the asymptotic limit. This increase in the density of available eigenmodes as a function of period is consistent with the increase we find in the number of observed independent modes, when comparing the hotter ZZ Ceti stars to the cooler ZZ Ceti stars.

\subsection{Observed Pulsation Periods}

Pulsations in ZZ Ceti models (and in stars in general) are selfdriven oscillations. Stochastic noise frequencies coincident with the eigenfrequencies are amplified by the driving mechanism to observable amplitudes. The blue edge of the ZZ Ceti instability strip occurs when the star is cool enough to have a hydrogen partial ionization zone sufficiently deep to excite global pulsations.

Our models suggest that the driving frequency is governed by the thermal timescale at the base of the hydrogen partial-ionization zone (see Cox 1980, p. 393; Unno et al. 1989). As the model star cools, the base of the partial-ionization zone moves deeper in the model, and the thermal timescale increases (Winget 1982; Hansen et al. 1985). Numerical calculations by Montgomery (2005) show that the thermal timescale at the base of the ionization zone $\tau_{\text {th }}$ is proportional to $T_{\text {eff }}^{-90}$. However, recent investigations by Kim et al. (2005) suggest that the thermal timescale is not consistent with the mean pulsation period, although both quantities increase in the models as we traverse from the blue to the red edge of the instability strip.

Figure 1 shows the mean pulsation period as a function of effective temperature for DAVs in both the SDSS and BG04 ZZ Ceti samples. We determine the weighted mean period (WMP) for each DAV by weighting each period with the corresponding observed amplitude,

$$
\mathrm{WMP}=\frac{\sum_{i} P_{i} A_{i}}{\sum_{i} A_{i}} .
$$

We can now appreciate the significance of Figure 1; the weighted mean pulsation period provides us with a method of determining the temperature of a ZZ Ceti star independent of spectroscopy. The respective equations of the best fit lines through the SDSS and the BG04 ZZ Ceti samples shown in Figure 1 are $\mathrm{WMP}_{\text {SDSS }}=-0.835^{ \pm 0.089} T_{\text {eff SDSS }}+10,060^{ \pm 1020}$ and $\mathrm{WMP}_{\mathrm{BG} 04}=-0.830^{ \pm 0.079} T_{\text {eff.BG04 }}+10,240^{ \pm 920}$, where WMP is in units of seconds, and $T_{\text {eff }}$ is in units of kelvin. It is reassuring to see an agreement in the values of the slope for both the SDSS and BG04 ZZ Ceti samples, and this gives us more confidence in using this period-temperature correlation as a means to measure the effective temperature of a DAV. We can now determine a temperature for the five variables with unreliable spectroscopic fits we excluded earlier, HS 0951+ 1312, HS 0952+1816, WD 1443+0134, WD 1524-0030, and WD 2350-0054 (see Table 1).

We compute a sum of the squares of the horizontal offsets of the points in Figure 1 from the above best-fit lines and arrive at a seismic estimate of the average uncertainty in our spectroscopic temperature. We determine $\sigma_{T_{\text {eff }}}=188.3 \mathrm{~K}$ for the SDSS ZZ Ceti sample and $\sigma_{T_{\text {eff }}}=185.4 \mathrm{~K}$ for the BG04 ZZ Ceti sample. It is not surprising that the average $T_{\text {eff }}$ uncertainties in these two samples turned out to be similar, because ZZ Ceti stars in both samples define an instability strip of the same width, $\sim 1000$ $1200 \mathrm{~K}$ (Mukadam et al. 2004a; Gianninas et al. 2005). We can expect that if the average $T_{\text {eff }}$ uncertainty in the SDSS sample had been significantly larger than that of the BG04 sample, the empirical width of the SDSS instability strip would have been correspondingly larger.

Our current temperature determinations are getting better and may be internally consistent to about $200 \mathrm{~K}$, at present. Our empirical measure of the width of the instability strip appears to have reduced from $1500 \mathrm{~K}$ (Bergeron et al. 1995; Koester \& Allard 2000) to about 1000-1200 K (Mukadam et al. 2004a; Gianninas et al. 2005). But even with our current atmospheric models, we do find exceptions to this period- $T_{\text {eff }}$ correlation, for which the spectroscopic temperature does not match the pulsation properties of the ZZ Ceti star. For example, Mukadam et al. (2004b, 2004a) report the ZZ Ceti star WD 2350-0054 with a dominant period close to $300 \mathrm{~s}$ as an unusual pulsator, because its SDSS spectroscopic determination places it below the red edge of the instability strip at $T_{\text {eff }}=10,350 \pm 60 \mathrm{~K}$. Bergeron et al. (2004) report a known complex pulsator G29-38 (Kleinman et al. 1998) as a hot ZZ Ceti star with $T_{\text {eff }}=11,820 \mathrm{~K}$. Better signal-to-noise ratio $(\mathrm{S} / \mathrm{N})$ spectra of these and other such ZZ Ceti stars may reveal temperatures consistent with their observed periods. Unless the pulsation characteristics themselves reveal curious features, it seems premature to conclude that the ZZ Ceti star in question (with a spectroscopic temperature inconsistent with its pulsation spectrum) is strange or abnormal in any way.

\subsubsection{Weighted Mean Period as a Temperature Indicator}

Measuring the pulsation periods of a ZZ Ceti star is a modelindependent and straightforward process, ${ }^{3}$ unlike the spectroscopic determination of its temperature. The instability strip spans $1000-1200 \mathrm{~K}$ in temperature and about $1300 \mathrm{~s}$ in period. The internal uncertainty in measuring temperature using spectra is typically about $200 \mathrm{~K}$, which is $17 \%-20 \%$ of the width of the instability strip. While even low-quality photometric data will yield a period precise to at least a few seconds. For the hotter ZZ Ceti stars, with little or no amplitude modulation, the uncertainty in WMP constitutes less than $1 \%$ of the width of the period-based instability strip. Cooler ZZ Ceti stars with a significant amplitude modulation can exhibit pulsation spectra with a WMP different by $30-60 \mathrm{~s}$ and, in a few cases, even as much as $100-200 \mathrm{~s}$. For most of the cooler ZZ Ceti stars, the uncertainty in WMP represents less than 5\% of the width of the

\footnotetext{
3 While it is true that aliases complicate the determination of pulsation periods from single site data, this ambiguity may change the value of the period typically by only a few seconds.
} 
TABLE 1

Pulsation Spectra of the $41+5$ ZZ Ceti Stars Mainly from the SDSS ZZ Ceti Sample

\begin{tabular}{|c|c|c|c|c|}
\hline Object & Class & $T_{\text {eff }}(\mathrm{K})$ & $\log g$ & $\begin{array}{l}\text { Linearly Independent Modes } \\
\text { Period (s)/Amplitude (mma) }\end{array}$ \\
\hline \multicolumn{5}{|c|}{ SDSS DAVs } \\
\hline WD $0018+0031^{\mathrm{a}} \ldots \ldots \ldots \ldots \ldots$ & hDAV & 11700 & 7.93 & $257.9 / 5.8$ \\
\hline WD $0048+1521^{\mathrm{a}} \ldots \ldots \ldots \ldots \ldots$ & iDAV & 11290 & 8.23 & $615.3 / 24.8$ \\
\hline WD $0102-0032 \ldots \ldots \ldots \ldots . . . .$. & cDAV & 11050 & 8.24 & $1043.4 / 15.3,926.3 / 34.7,830.3 / 35.1,752.2 / 19.4$ \\
\hline WD $0111+0018 \ldots \ldots \ldots \ldots \ldots . .$. & hDAV & 11510 & 8.26 & $292.3 / 21.9,255.3 / 15.6$ \\
\hline WD $0214-0823 \ldots \ldots \ldots \ldots . . .$. & hDAV & 11570 & 7.92 & $348.1 / 8.4,347.1 / 8.2,297.5 / 16.0,263.5 / 7.1$ \\
\hline WD $0318+0030 \ldots \ldots \ldots \ldots \ldots$ & cDAV & 11040 & 8.07 & $844.9 / 15.3,826.4 / 27.3,695.0 / 8.9,587.1 / 10.6,536.1 / 11.1$ \\
\hline WD $0332-0049 \ldots \ldots \ldots \ldots . . .$. & cDAV & 11040 & 8.25 & $1143.7 / 7.4,938.4 / 6.7,765.0 / 15.2,402.0 / 4.1$ \\
\hline WD $0332-0049$.............. & cDAV & 11040 & 8.25 & $770 / 23,910 / 10$ \\
\hline WD $0756+2020^{\mathrm{a}} \ldots \ldots \ldots \ldots . . .$. & hDAV & 11710 & 8.01 & $199.5 / 6.8$ \\
\hline 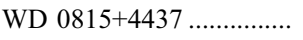 & iDAV & 11620 & 7.93 & $787.5 / 7.2,311.7 / 18.9,309.0 / 10.2,258.3 / 6.8$ \\
\hline WD $0818+3131^{\mathrm{a}} \ldots \ldots \ldots \ldots \ldots$ & hDAV & 11800 & 8.07 & $253.3 / 2.9,202.3 / 3.3$ \\
\hline WD $0825+4119 \ldots \ldots \ldots \ldots \ldots$ & iDAV & 11820 & 8.49 & $653.4 / 17.1,611.0 / 11.2$ \\
\hline WD $0842+3707 \ldots \ldots \ldots \ldots \ldots . . . .$. & hDAV & 11720 & 7.73 & $321.1 / 4.4,309.3 / 18.0,212.3 / 5.2$ \\
\hline WD $0847+4510 \ldots \ldots \ldots \ldots . . . .$. & hDAV & 11680 & 8.00 & $200.5 / 7.0,123.4 / 3.0$ \\
\hline WD $0906-0024 \ldots \ldots \ldots \ldots . . . .$. & iDAV & 11520 & 8.00 & $769.4 / 26.1,618.8 / 9.3,574.5 / 23.7,457.9 / 9.8,266.6 / 7.6$ \\
\hline WD $0913+4036^{\mathrm{a}} \ldots \ldots \ldots \ldots \ldots$ & hDAV & 11680 & 7.87 & $320.5 / 14.7,288.7 / 12.4,260.3 / 16.5,203.9 / 3.8$ \\
\hline 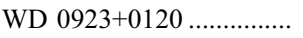 & cDAV & 11150 & 8.74 & $655.7 / 4.4$ \\
\hline 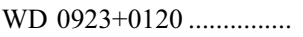 & cDAV & 11150 & 8.74 & $668.9 / 3.5$ \\
\hline 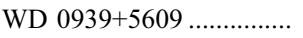 & hDAV & 11790 & 8.22 & $249.9 / 7.2,48.5 / 5.9$ \\
\hline WD $0942+5733 \ldots \ldots \ldots \ldots \ldots$ & iDAV & 11260 & 8.27 & $909.4 / 7.7,694.7 / 37.7,550.5 / 12.3,273.0 / 9.0$ \\
\hline WD $0949-0000 \ldots \ldots \ldots \ldots . . .$. & iDAV & 11180 & 8.22 & $711.6 / 6.0,634.2 / 5.1,516.6 / 16.2,365.2 / 17.7,364.1 / 7.3,363.2 / 12.5$ \\
\hline WD $0958+0130 \ldots \ldots \ldots \ldots . . . .$. & hDAV & 11680 & 7.99 & $264.4 / 4.7,203.7 / 2.5,121.2 / 1.6$ \\
\hline WD $1002+5818^{\mathrm{a}} \ldots \ldots \ldots \ldots \ldots$ & hDAV & 11710 & 7.92 & $304.6 / 5.3,268.2 / 6.8$ \\
\hline WD $1007+5245^{\mathrm{a}} \ldots \ldots \ldots \ldots . .$. & hDAV & 11430 & 8.08 & $323.1 / 10.4,290.1 / 7.7,258.8 / 11.0$ \\
\hline 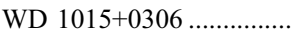 & hDAV & 11580 & 8.14 & $270.0 / 8.4,255.7 / 7.3,194.7 / 5.8$ \\
\hline 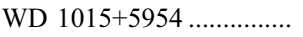 & iDAV & 11630 & 8.02 & $769.9 / 5.7,453.8 / 15.5,401.7 / 21.4,292.4 / 8.6$ \\
\hline 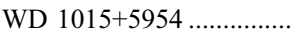 & iDAV & 11630 & 8.02 & $768.4 / 7.5,455.3 / 17.7,401.4 / 19.8,294.0 / 9.1$ \\
\hline 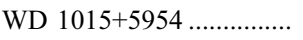 & iDAV & 11630 & 8.02 & $456.1 / 19.6,399.7 / 19.2,145.5 / 3.1,139.2 / 4.6$ \\
\hline WD $1054+5307^{\mathrm{a}} \ldots \ldots \ldots \ldots \ldots$ & cDAV & 11120 & 8.01 & $869.1 / 37.4$ \\
\hline WD $1056-0006 \ldots \ldots \ldots \ldots . . . .$. & cDAV & 11020 & 7.86 & $1024.9 / 31.6,925.4 / 60.3,670.6 / 12.0,603.0 / 11.5$ \\
\hline WD $1122+0358 \ldots \ldots \ldots \ldots \ldots . .$. & cDAV & 11070 & 8.06 & $996.1 / 17.3,859.1 / 34.6,740.1 / 10.0$ \\
\hline 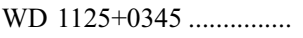 & hDAV & 11600 & 7.99 & $335.1 / 2.8,265.8 / 3.3,265.5 / 7.1,208.6 / 2.8$ \\
\hline 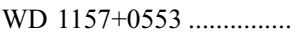 & cDAV & 11050 & 8.15 & $1056.2 / 5.8,918.9 / 15.9,826.2 / 8.1,748.5 / 5.6$ \\
\hline WD $1345-0055 \ldots \ldots \ldots \ldots . .$. & hDAV & 11800 & 8.04 & $254.4 / 2.4,195.5 / 3.9,195.2 / 5.5$ \\
\hline 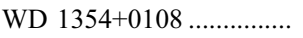 & hDAV & 11700 & 8.00 & $322.9 / 1.9,291.6 / 2.2,198.3 / 6.0,173.3 / 1.1,127.8 / 1.5$ \\
\hline WD $1355+5454^{\mathrm{a}} \ldots \ldots \ldots \ldots . .$. & hDAV & 11580 & 7.95 & $324.0 / 21.8$ \\
\hline WD $1417+0058 \ldots \ldots \ldots \ldots \ldots . .$. & cDAV & 11300 & 8.04 & $980.0 / 11.3,894.6 / 42.8,812.5 / 32.1,749.4 / 17.9,522.0 / 14.9$ \\
\hline WD $1502-0001 \ldots \ldots \ldots \ldots . . . .$. & iDAV & 11200 & 8.00 & $687.5 / 12.0,629.5 / 32.6,581.9 / 11.1,418.2 / 14.9$ \\
\hline WD $1617+4324 \ldots \ldots \ldots \ldots \ldots$ & cDAV & 11190 & 8.03 & $889.7 / 36.6,661.7 / 21.2,626.3 / 15.4$ \\
\hline WD $1700+3549 \ldots \ldots \ldots \ldots \ldots$ & cDAV & 11160 & 8.04 & $1164.4 / 11.4,955.3 / 20.3,893.4 / 54.3,552.6 / 9.3$ \\
\hline 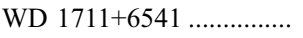 & cDAV & 11310 & 8.64 & $1248.2 / 3.2,690.2 / 3.3,606.3 / 5.2,234.0 / 1.2$ \\
\hline WD $1711+6541 \ldots \ldots \ldots \ldots . . .$. & cDAV & 11310 & 8.64 & $1186.6 / 3.3,934.8 / 2.9,612.6 / 5.7,561.5 / 3.0,214.3 / 1.7$ \\
\hline 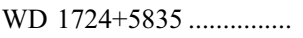 & hDAV & 11540 & 7.89 & $337.9 / 5.9,279.5 / 8.3,189.2 / 3.2$ \\
\hline 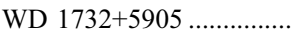 & cDAV & 10860 & 7.99 & $1248.4 / 4.5,1122.4 / 8.0$ \\
\hline 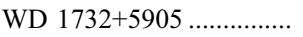 & cDAV & 10860 & 7.99 & $1336 / 7.8,1090 / 8.0$ \\
\hline WD $2159+1322^{\mathrm{a}} \ldots \ldots \ldots \ldots \ldots$ & cDAV & 11710 & 8.61 & $801.0 / 15.1,683.7 / 11.7$ \\
\hline WD $2214-0025^{\mathrm{a}} \ldots \ldots \ldots \ldots . .$. & hDAV & 11440 & 8.33 & $255.2 / 13.1,195.2 / 6.1$ \\
\hline \multicolumn{5}{|c|}{ Five DAVs with Unreliable Spectroscopic Fits } \\
\hline 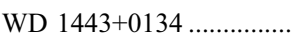 & cDAV & $10830^{\mathrm{b}}$ & $\ldots$ & $1085.0 / 5.2,969.0 / 7.5$ \\
\hline WD $1524-0030 \ldots \ldots \ldots \ldots . . .$. & cDAV & $11160^{\mathrm{b}}$ & $\ldots$ & $873.3 / 110.8,717.5 / 28.3,498.6 / 21.6,255.2 / 17.9$ \\
\hline WD $2350-0054 \ldots \ldots \ldots \ldots . . .$. & hDAV & $11690^{\mathrm{b}}$ & $\ldots$ & $391.1 / 3.1,304.1 / 16.3,272.8 / 16.2$ \\
\hline WD $2350-0054 \ldots \ldots \ldots \ldots \ldots$ & hDAV & $11710^{\mathrm{b}}$ & $\ldots$ & $304.5 / 13.8,272.7 / 14.8,212.6 / 3.0$ \\
\hline WD $2350-0054 \ldots \ldots \ldots \ldots . . . .$. & hDAV & $11680^{\mathrm{b}}$ & $\ldots$ & $391.1 / 7.5,304.3 / 17.0,273.3 / 6.3,206.7 / 3.2$ \\
\hline 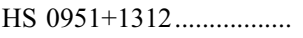 & hDAV & $11740^{\mathrm{b}}$ & $\ldots$ & $311.7 / 2.7,282.2 / 9.0,258.0 / 3.6,208.0 / 9.4$ \\
\hline 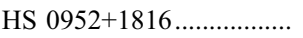 & cDAV & $10800^{\mathrm{b}}$ & $\ldots$ & $1160.9 / 7.9,945.9 / 10.4$ \\
\hline 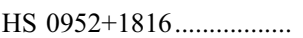 & cDAV & $10960^{\mathrm{b}}$ & $\ldots$ & $1150 / 4.8,883 / 3.6,790 / 2.9,674.7 / 3.0$ \\
\hline
\end{tabular}

${ }^{\mathrm{a}}$ We obtained the pulsation spectrum from Mullally et al. (2005).

$\mathrm{b}$ Temperatures derived using the best fit to the weighted mean period-spectroscopic temperature plot (Fig. 1, top panel) using the first 41 SDSS DAVs listed in this Table. 
instability strip. ${ }^{4}$ We therefore expect that this method of using the WMP to effectively measure the location of a DAV within the instability strip is, in general, more accurate and reliable than spectroscopy.

Any dispersion in period due to differences in stellar mass, core composition, H/He layer masses, etc., can increase the uncertainty of determining the temperature of a given DAV using this relation. We expect this may be significantly smaller than our current typical spectroscopic $T_{\text {eff }}$ uncertainties of a few $100 \mathrm{~K}$.

Note that we do not claim that the relationship between the WMP and the temperature is linear; a straight line is merely the simplest model fit possible to the observations shown in Figure 1, considering the large amount of scatter. We do not necessarily require a linear relation between WMP and $T_{\text {eff }}$ for this method to work as a temperature indicator. Our collaborators at the University of Texas are currently investigating the interpretation of these data in terms of the theoretical models (A. Kim et al. 2006, in preparation).

The plot of WMP versus $T_{\mathrm{eff}}$ is similar to comparing two independent temperature scales, each with its own independent source of uncertainties. The uncertainty in spectroscopic $T_{\text {eff }}$ depends on the quality of the spectrum, the accuracy and completeness of the model atmosphere, and the details of the algorithm used to fit the observed spectrum with the template spectra. Large-amplitude variables have a corresponding higher uncertainty in temperature (McGraw 1979). The uncertainty in WMP comes from the quality of the photometric observations and the amplitude modulation of the star.

As long as we restrict our relative $T_{\text {eff }}$ parameter in units of seconds in the WMP temperature scale, the uncertainty in our measurements is not related to spectroscopic temperature values at all. It is only when we attempt to translate WMP into a temperature in degrees kelvin that we have to use the relation between WMP and spectroscopic temperature. Even in this case, we are still better off than the typical $200 \mathrm{~K}$ uncertainty in spectroscopic temperature, because the slopes of the best-fit lines in Figure 1 depend not on a few stars but on 35-40 stars. Using WMP directly as a temperature scale is nonintuitive at present, but this may be worth thinking about as an alternative scale in the long run, once we improve our understanding of the relation between WMP and $T_{\text {eff, }}$, both observationally and theoretically.

\subsubsection{Redefining the ZZ Ceti Classification}

We currently classify the ZZ Ceti stars into two groups, the hot DAVs and the cool DAVs. However, a clear dividing point (in temperature) between these two classes does not exist to date in published literature. Our present classification of these stars is thus vague in this context, although it has certainly proved to be a useful guide. The primary reason such a temperature-based classification cannot be well defined is that the location and width of the instability strip are model-dependent features. Model atmospheres of DAV stars treat convection with some parameterization, the choice of which can shift the instability strip in temperature by a few thousand K (Bergeron et al. 1995; Koester \& Allard 2000).

The pulsation characteristics of the ZZ Ceti stars helped us divide them into simple and complex pulsators in the late 1970's.

\footnotetext{
${ }^{4}$ G29-38 is the only example we find in the literature where the published pulsation spectra exhibit a change in WMP by $375 \mathrm{~s}$ (see McGraw \& Robinson 1975; Kleinman 1995; Kleinman et al. 1998). It is a complex pulsator with a spectroscopic temperature of a hot ZZ Ceti star (Bergeron et al. 2004); its large amplitude probably indicates a temperature excursion of up to $500 \mathrm{~K}$, a substantial part of the instability strip. It seems difficult to determine a reliable effective temperature of G29-38 by either method.
}

A decade later, we recognized that the simple pulsators that exhibit a few modes with short periods (200-300 s), small amplitudes (few mma), sinusoidal or sawtooth pulse shapes, and continued to show the same pulsation spectra over a few decades, were hot ZZ Ceti stars. We also realized that the complex pulsators that exhibit several long periods (600-1200 s) with large amplitudes (40-110 mma), nonsinusoidal pulse shapes with fast rise times and slow decadence, and amplitude modulation over timescales of a few days to a few years, were cool ZZ Ceti stars. Although we now use spectroscopic temperature as a means to classify these stars, the classification scheme came about only because we initially used the pulsation characteristics to separate these stars into two groups.

We suggest a new ZZ Ceti classification scheme based on the WMP of these variables. We intend to retain the fundamental aspect of the previous scheme in using a temperature-based classification. We have shown that WMP is also a temperature scale (Fig. 1). We expect that WMP serves as a more accurate and reliable $T_{\text {eff }}$ scale than spectroscopic temperature, because measuring the pulsation periods of a ZZ Ceti star constitutes a relatively simple, less uncertain, and model-independent exercise compared to measuring its spectroscopic temperature.

We redefine the class of hot DAVs (hDAVs) as ZZ Ceti stars with WMP $<350 \mathrm{~s}$. We redefine the class of cool DAVs (cDAVs) as ZZ Ceti stars with WMP > $650 \mathrm{~s}$. We suggest introducing a new class of DAVs, to be called the intermediate DAVs (iDAVs), as ZZ Ceti stars with $350 \leq \mathrm{WMP} \leq 650$. This class merely forms the evolutionary subclass adjoining the hot and cool ZZ Ceti stars and typically encompasses those ZZ Ceti pulsators that show a large range of pulsation periods, e.g., HS 0507+0435B.

For borderline cases such as G29-38, when one season of observations place the DAV in one class and a second season places it in another class, then we suggest choosing the cooler class of the two possibilities. We find several cases in which a cDAV or an iDAV exhibit modes typical of the hotter pulsators, but we have not seen an instance of a hot DAV exhibiting a mode typical of the cool ZZ Ceti stars. We show our suggested classification for most of the known noninteracting ZZ Ceti stars in Tables 1 and 2.

Note that the boundaries of 350 and $650 \mathrm{~s}$ are arbitrary in some sense; we merely wished to divide the instability strip into three parts and used a period histogram to fine-tune our choice. For simplicity and better readability, Tables 1 and 2 do not show all of the pulsation spectra we accrued for each star. We show multiple seasons of observations only for those DAVs that exhibit different frequencies and amplitudes at different times. This also helps us understand the intrinsic changes in weighted mean period and mean pulsation amplitude for such variables. To use such stars in the ensemble, we computed the weighted mean period and mean pulsation amplitude for each season of observations individually, and then included the average value in our analysis. We refer the reader to Silvotti et al. (2005, 2006), Castanheira et al. (2005), Kepler et al. (2005), and Gianninas et al. (2005) for the pulsation spectra of the new DAVs.

\subsection{Observed Pulsation Amplitudes}

The physical quantity of interest in this subsection is the intrinsic pulsation amplitude of modes excited in the ZZ Ceti star. However, the measured amplitudes of observed modes are most likely lower than the intrinsic amplitudes due to geometric cancellation. This effect has three independent causes: disk averaging, inclination angle, and limb darkening. We are unable to resolve the disk of the star from Earth. Hence, the observed amplitude of each pulsation mode is lower due to a disk-averaging 
TABLE 2

Pulsation Spectra of the 36 ZZ Ceti Stars in the BG04 ZZ Ceti Sample

\begin{tabular}{|c|c|c|c|c|c|}
\hline Object & Class & $T_{\text {eff }}(\mathrm{K})$ & $\log g$ & $\begin{array}{l}\text { Linearly Independent Modes } \\
\text { Period (s)/Amplitude (mma) }\end{array}$ & Reference \\
\hline 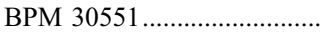 & $\mathrm{cDAV}$ & 11260 & 8.23 & $920.5 / 18.5,741.4 / 21.7,655.4 / 17.4,442.8 / 6.5$ & Hesser et al. (1976) \\
\hline 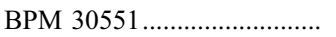 & $\mathrm{cDAV}$ & 11260 & 8.23 & $993.0 / 6.5,936.2 / 13.0,885.6 / 15.2,819.2 / 19.5,738.0 / 6.5,609.6 / 6.5$ & Hesser et al. (1976) \\
\hline 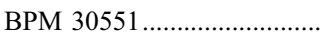 & $\mathrm{cDAV}$ & 11260 & 8.23 & $963.8 / 13.0,844.5 / 19.5,799.2 / 10.9,682.7 / 13.0,606.8 / 13.0$ & Hesser et al. (1976) \\
\hline 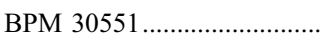 & cDAV & 11260 & 8.23 & $920.4 / 8.7,751.6 / 13.0,682.7 / 10.9,606.8 / 15.2,546.1 / 5.4,496.5 / 4.3$ & Hesser et al. (1976) \\
\hline 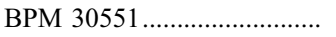 & cDAV & 11260 & 8.23 & $910.2 / 8.7,862.3 / 6.5,731.4 / 6.5,682.7 / 13.0,607.9 / 17.4$ & Hesser et al. (1976) \\
\hline 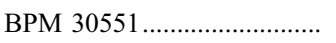 & cDAV & 11260 & 8.23 & $1129.9 / 10.9,1057.0 / 8.7,744.7 / 18.5,606.8 / 16.3$ & Hesser et al. (1976) \\
\hline BPM $30551 \ldots \ldots \ldots \ldots \ldots \ldots \ldots \ldots . .$. & $\mathrm{cDAV}$ & 11260 & 8.23 & $1137.8 / 8.7,958.1 / 7.6,862.3 / 16.3,744.7 / 7.6,606.8 / 14.1$ & Hesser et al. (1976) \\
\hline BPM $30551 \ldots \ldots \ldots \ldots \ldots \ldots \ldots \ldots \ldots$ & $\mathrm{cDAV}$ & 11260 & 8.23 & $1092.3 / 5.4,993.0 / 8.7,936.2 / 9.8,744.7 / 7.6,712.3 / 6.5,606.8 / 11.9$ & Hesser et al. (1976) \\
\hline 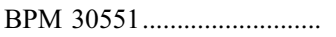 & cDAV & 11260 & 8.23 & $862.3 / 6.5,799.2 / 8.7,744.7 / 7.6,668.7 / 7.6,612.5 / 5.4$ & Hesser et al. (1976) \\
\hline 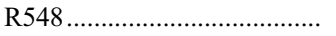 & $\mathrm{hDAV}$ & 11990 & 7.97 & $333.6 / 0.6,318.0 / 0.9,274.8 / 3.8,274.3 / 4.8,213.1 / 7.4,212.8 / 4.7,187.3 / 0.9$ & Mukadam et al. (2003) \\
\hline МCT $0145-2211$.................... & iDAV & 11550 & 8.14 & $823.2 / 17,727.9 / 19,462.2 / 25$ & Fontaine et al. (2003) \\
\hline KUV $02464+3239 \ldots \ldots \ldots \ldots \ldots . . .$. & $\mathrm{cDAV}$ & 11290 & 8.08 & $839.6 / 39$ & Fontaine et al. (2001) \\
\hline 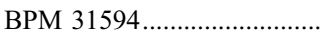 & iDAV & 11540 & 8.11 & $617.9 / 48.0,401.6 / 16,416.1 / 5$ & O’Donoghue et al. (1992) \\
\hline HL Tau 76 & iDAV & 11450 & 7.89 & $933 / 25.2,796 / 9.7,781 / 9.9,541 / 40.5,494 / 30.2,383 / 21.8$ & Dolez (1998) \\
\hline 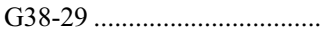 & cDAV & 11180 & 7.91 & $1024.0 / 26.1,938.0 / 26.5$ & McGraw \& Robinson (1975) \\
\hline 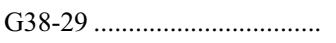 & cDAV & 11180 & 7.91 & $1019.8 / 12.2,910.3 / 28.3$ & McGraw \& Robinson (1975) \\
\hline G191-16 ............................... & $\mathrm{cDAV}$ & 11420 & 8.05 & $892.9 / 100$ & Vauclair et al. (1989) \\
\hline 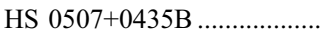 & iDAV & 11630 & 8.17 & 743.0/13.9, 557.7/18.7, 446.2/11.0, 444.8/13.6, 355.8/22.7, 354.9/6.8, 286.1/3.6 & Kotak et al. (2002) \\
\hline HS $0507+0435 B \ldots \ldots \ldots \ldots \ldots \ldots . . . .$. & iDAV & 11630 & 8.17 & $\begin{array}{l}\text { 743.4/8.3, 588.7/3.9, 583.8/1.6, 559.6/2.7, 557.6/17.4, 557.2/3.1, 556.5/7.8, 555.3/18.0, } \\
\quad 446.1 / 15.1,445.3 / 3.0,444.6 / 12.9,355.8 / 26.1,355.4 / 4.7,354.9 / 11.1\end{array}$ & Handler et al. (2002) \\
\hline 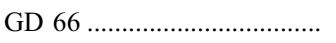 & hDAV & 11980 & 8.05 & $649.4 / 2.2,441.9 / 1.6,301.7 / 6.7,271.7 / 8.4,196.5 / 3.6$ & Fontaine et al. (1985) \\
\hline GD $66 \ldots \ldots \ldots \ldots \ldots \ldots \ldots \ldots \ldots \ldots \ldots \ldots$ & $\mathrm{hDAV}$ & 11980 & 8.05 & $788.6 / 5.5,461.0 / 3.6,301.7 / 6.3,271.7 / 30,197.2 / 2.2$ & Fontaine et al. (1985) \\
\hline GD $66 \ldots \ldots \ldots \ldots \ldots \ldots \ldots \ldots \ldots \ldots \ldots \ldots$ & $\mathrm{hDAV}$ & 11980 & 8.05 & $304.5 / 8.8,271.1 / 14.8,256.5 / 9,197.8 / 7,123.1 / 2.3$ & Fontaine et al. (2001) \\
\hline 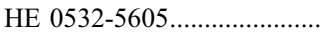 & iDAV & 11560 & 8.49 & $688.8 / 8.3,586.4 / 7.9$ & Fontaine et al. (2003) \\
\hline KUV $08368+4026 \ldots \ldots \ldots \ldots . . .$. & iDAV & 11490 & 8.05 & $618.0 / 17.4,494.5 / 6.0$ & Vauclair et al. (1997) \\
\hline 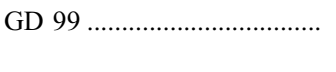 & $\mathrm{cDAV}$ & 11820 & 8.08 & $\begin{array}{l}1151.0 / 1.9,1088.0 / 4.3,1058.0 / 8.3,1007.0 / 6.5,976.0 / 2.1,924.7 / 1.7,853.2 / 2.4,633.1 / 2.0 \\
\quad 228.9 / 4.5,223.6 / 2.9,105.2 / 2.0\end{array}$ & $\begin{array}{l}\text { K. M. Chynoweth \& S. Thompson } \\
\text { (2005, private communication) }\end{array}$ \\
\hline G117-B15A …….................... & hDAV & 11630 & 7.97 & $304.4 / 8.2,271.0 / 7.3,215.2 / 23.9$ & Kepler et al. (1982) \\
\hline KUV $11370+4222 \ldots \ldots \ldots \ldots \ldots . .$. & $\mathrm{hDAV}$ & 11890 & 8.06 & $462.9 / 3.5,292.2 / 2.7,257.2 / 5.8$ & Vauclair et al. (1997) \\
\hline G255-2 ………………...... & cDAV & 11440 & 8.17 & $898.5 / 9.4,855.4 / 11.2,819.7 / 11.3,775.2 / 15.2,681.2 / 24.9,607.9 / 13.1,568.5 / 6.6$ & G. Vauclair (2005, private communication) \\
\hline 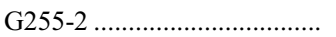 & $\mathrm{cDAV}$ & 11440 & 8.17 & $985.2 / 4.8,773.4 / 12.7,681.2 / 27.7,568.5 / 16.5$ & G. Vauclair (2005; private communication) \\
\hline 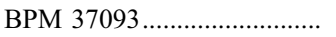 & iDAV & 11730 & 8.81 & $636.7 / 1.7,633.2 / 1.1,613.5 / 1.1,600.7 / 0.9,582.0 / 1.0,565.5 / 1.2,562.6 / 0.9,548.4 / 1.1$ & Kanaan et al. (2005) \\
\hline 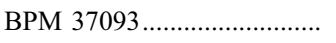 & iDAV & 11730 & 8.81 & $660.8 / 0.5,637.2 / 0.7,633.5 / 1.3,565.9 / 0.5,549.2 / 0.8,531.1 / 1.2,511.7 / 0.7$ & Kanaan et al. (2005) \\
\hline 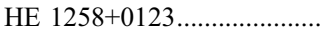 & cDAV & 11410 & 8.04 & $1092.1 / 14,744.6 / 23,528.5 / 9$ & Bergeron et al. (2004) \\
\hline 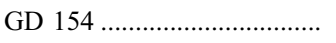 & cDAV & 11180 & 8.15 & $1190.5 / 6.3,1186.5 / 16.7,1183.5 / 4.6,1092.1 / 3.0,1088.6 / 5.0,1084.0 / 5.6$ & Pfeiffer et al. (1995) \\
\hline LP $133-144 \ldots \ldots \ldots \ldots \ldots \ldots \ldots \ldots \ldots .$. & $\mathrm{hDAV}$ & 11800 & 7.87 & $327.3 / 4,306.9 / 4,304.5 / 4,209.2 / 10$ & Bergeron et al. (2004) \\
\hline $\mathrm{G} 238-53 \ldots \ldots \ldots \ldots \ldots \ldots \ldots \ldots \ldots \ldots \ldots$ & $\mathrm{hDAV}$ & 11890 & 7.91 & $206.2 / 8$ & Fontaine \& Wesemael (1984) \\
\hline
\end{tabular}


TABLE 2-Continued

\begin{tabular}{|c|c|c|c|c|c|}
\hline Object & Class & $T_{\text {eff }}(\mathrm{K})$ & $\log g$ & $\begin{array}{l}\text { Linearly Independent Modes } \\
\text { Period (s)/Amplitude (mma) }\end{array}$ & Reference \\
\hline 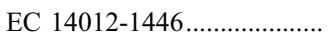 & iDAV & 11900 & 8.16 & $937 / 11,610 / 57,724 / 21,530 / 15,399 / 13$ & Stobie et al. (1995) \\
\hline GD 165 & $\mathrm{hDAV}$ & 11980 & 8.06 & $\begin{array}{l}249.7 / 0.7,192.8 / 0.9,192.7 / 2.4,192.6 / 1.9,166.2 / 0.4,146.4 / 0.5,120.4 / 1.8,120.36 / 4.8 \\
\quad 120.3 / 1.4,114.3 / 0.6,107.7 / 0.4\end{array}$ & Bergeron et al. (1993) \\
\hline L19-2 ............................... & $\mathrm{hDAV}$ & 12100 & 8.21 & $\begin{array}{l}350.1 / 1.1,348.7 / 0.5,192.6 / 6.5,193.1 / 0.9,192.1 / 0.8,143.4 / 0.6,143.0 / 0.3,118.9 / 0.3,118.7 / 1.2,118.5 / 2.0 \\
\quad 114.2 / 0.3,113.8 / 2.4,113.3 / 0.6\end{array}$ & O’Donoghue \& Warner (1982) \\
\hline PG $1541+651 \ldots \ldots \ldots \ldots \ldots \ldots \ldots \ldots$ & $\mathrm{cDAV}$ & 11600 & 8.10 & $757 / 14,689 / 45,564 / 15,467 / 3$ & Vauclair et al. (2000) \\
\hline 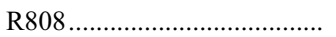 & $\mathrm{cDAV}$ & 11160 & 8.04 & $833 / 81$ & McGraw \& Robinson (1976) \\
\hline 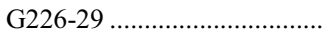 & hDAV & 12270 & 8.28 & $109.5 / 2.8,109.3 / 1.1,109.1 / 2.5$ & Kepler et al. (1995) \\
\hline 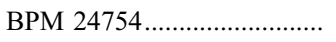 & cDAV & 11070 & 8.03 & $1176 / 22.6$ & Giovannini et al. (1998) \\
\hline 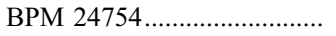 & cDAV & 11070 & 8.03 & 1050/9.1 & Giovannini et al. (1998) \\
\hline 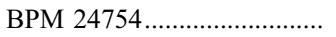 & cDAV & 11070 & 8.03 & $1086 / 13.2$ & Giovannini et al. (1998) \\
\hline 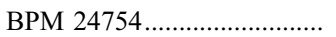 & $\mathrm{cDAV}$ & 11070 & 8.03 & $978 / 7.7$ & Giovannini et al. (1998) \\
\hline 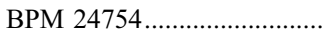 & cDAV & 11070 & 8.03 & 1098/6.1 & Giovannini et al. (1998) \\
\hline 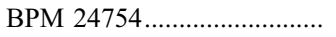 & cDAV & 11070 & 8.03 & $1122 / 6.7$ & Giovannini et al. (1998) \\
\hline G207-9 ............................. & iDAV & 11950 & 8.35 & $557.4 / 6.3,318.0 / 6.4,292.0 / 5.0,259.1 / 1.7$ & Robinson \& MacGraw (1976) \\
\hline G185-32 ……....................... & $\mathrm{hDAV}$ & 12130 & 8.05 & $651.7 / 0.7,537.6 / 0.6,454.6 / 0.4,370.2 / 1.6,301.4 / 1.1,299.8 / 1.0,264.2 / 0.5,215.7 / 1.9,141.9 / 1.4,72.9 / 0.4$ & Castanheira et al. (2004) \\
\hline G185-32 ……….................... & $\mathrm{hDAV}$ & 12130 & 8.05 & $370.2 / 1.3,301.6 / 1.5,215.7 / 1.9,141.9 / 1.5,72.6 / 0.7$ & Thompson et al. (2004) \\
\hline GD 385 & $\mathrm{hDAV}$ & 11710 & 8.04 & $256.3 / 10.9,256.1 / 11.4$ & Kepler (1984) \\
\hline 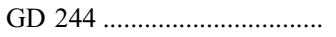 & $\mathrm{hDAV}$ & 11680 & 8.08 & $307.0 / 14,294.6 / 5.5,256.3 / 5,203.3 / 10.5$ & Fontaine et al. (2001) \\
\hline 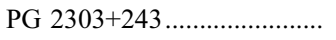 & cDAV & 11480 & 8.09 & $900.5 / 16,794.5 / 56,675.4 / 8,623.4 / 15,570.7 / 8$ & Vauclair et al. (1987) \\
\hline G29-38 ................................ & $\mathrm{cDAV}$ & 11820 & 8.14 & $1015.5 / 14.5,930.9 / 25.7,824.7 / 20.2,677.0 / 17.6,612.9 / 20.0$ & McGraw \& Robinson (1975) \\
\hline 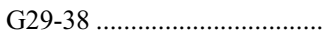 & $\mathrm{cDAV}$ & 11820 & 8.14 & $934.5 / 20.5,813.8 / 23.5,671.5 / 23.0,623.8 / 11.8$ & McGraw \& Robinson (1975) \\
\hline G29-38 ……........................ & cDAV & 11820 & 8.14 & $859.6 / 24.6,648.7 / 7.8,614.3 / 31.3,498.3 / 5.8,401.3 / 4.4,400.4 / 7.0,399.6 / 8.8,283.9 / 3.5$ & Kleinman (1995) \\
\hline 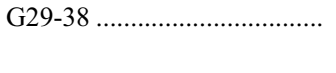 & cDAV & 11820 & 8.14 & $\begin{array}{l}\text { 915.4/5.9, 615.1/58.0, 500.4/8.0, 474.9/4.8, 401.3/5.6, 400.4/5.8, 399.6/10.0, 354.9/2.9, 333.9/2.7, } \\
\quad 283.9 / 3.5,110.1 / 0.9\end{array}$ & Kleinman (1995) \\
\hline 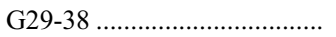 & $\mathrm{cDAV}$ & 11820 & 8.14 & $770.7 / 1.5,503.5 / 8.6,495.0 / 11.8,401.2 / 9.7,400.5 / 1.3,399.7 / 4.9,283.9 / 6.4,177.1 / 0.8$ & Kleinman (1995) \\
\hline 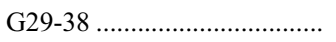 & cDAV & 11820 & 8.14 & $809.4 / 30.1,610.3 / 10.6,401.2 / 11.2,399.7 / 4.5,283.9 / 6.9$ & Kleinman (1995) \\
\hline G29-38 ............................... & cDAV & 11820 & 8.14 & $\begin{array}{l}\text { 894.0/14.0, 770.8/8.7, 678.4/9.7, 612.4/31.6, 610.7/8.2, 608.9/8.5, 551.9/4.4, 498.3/6.1, 401.2/6.0, } \\
\quad 399.7 / 5.7,237.0 / 1.8,236.4 / 1.8\end{array}$ & Kleinman (1995) \\
\hline 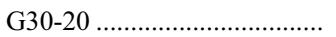 & $\mathrm{cDAV}$ & 11070 & 7.95 & $1068 / 13.8$ & Mukadam et al. (2002) \\
\hline 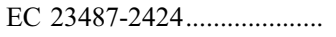 & cDAV & 11520 & 8.10 & $993.0 / 37.7,989.3 / 11.0,868.2 / 12.8,804.5 / 19.3$ & Stobie et al. (1993) \\
\hline
\end{tabular}




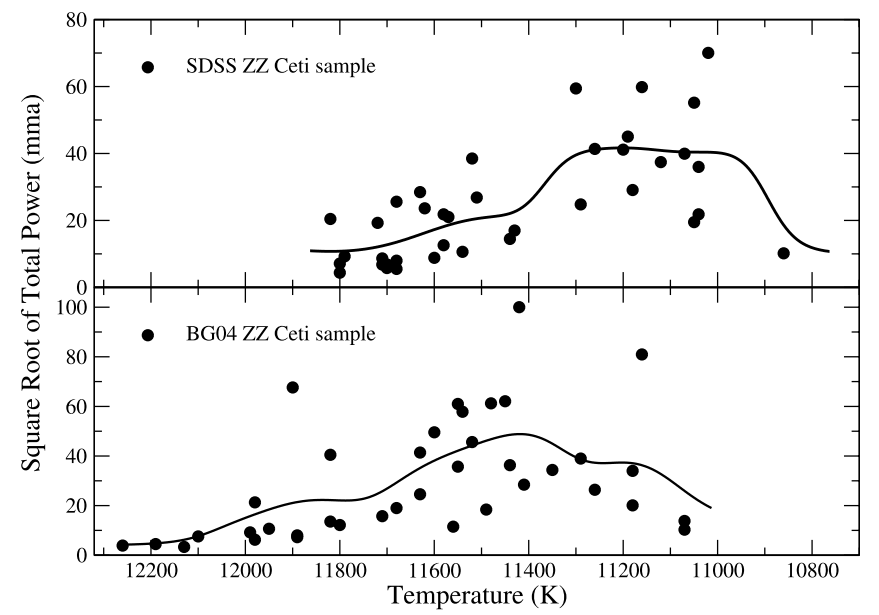

FIG. 2.-Square root of total power for 38 SDSS DAVs (top) and 38 DAVs from the BG04 sample (bottom) as a function of their spectroscopic temperature. The solid line shows a smooth curve obtained from Gaussian binning that demonstrates an initial increase in pulsation amplitude followed by a suggestive decline prior to the red edge.

effect. This explains why the probability of detecting $\ell=1$ modes is higher than the probability of detecting $\ell=2$ and $\ell=3$ modes. The inclination angle dictates the distribution of the bright and dark zones in our view for a given mode. Eigenmodes with different $m$ values exhibit different cancellation patterns (see Dziembowski 1977; Pesnell 1985).

Limb darkening effectively reduces the area of the stellar disk in our view, and this reduction in area depends on wavelength. At UV wavelengths, the increased limb darkening decreases the contribution of zones near the limb. As a result, modes of higher $\ell$ are canceled less effectively than low- $\ell$ modes in the UV (Robinson et al. 1982). Of these three independent causes of geometric cancellation, limb darkening is the only one that works in our favor, and provides us with a mode identification technique (Robinson et al. 1995).

The intrinsic pulsation amplitude depends on the mass of the star. Nonradial $g$-modes have a nonnegligible radial component, the amplitude of which scales with stellar mass and plays a role in dictating the amplitude of the nonradial component. The massive pulsators BPM $37093(\log g=8.81)$, WD $0923+0120$ $(\log g=8.74)$, WD $1711+6541(\log g=8.64)$, and WD $2159+$ $1322(\log g=8.61)$ exhibit low amplitudes as a result of their high gravity, and thus we exclude them from $\S 2.3$.

Figure 2 shows the square root of total power $\left(\Sigma_{i} A_{i}^{2}\right)^{1 / 2}$ for 38 stars from the SDSS sample (top) and 38 stars from the BG04 ZZ Ceti sample (bottom), plotted as a function of their spectroscopic temperature. All of the pulsation amplitudes we report in this paper come from optical whole-disk observations. We expect these are mostly low- $\ell$ modes. For these reasons, we expect them to be lower than the corresponding intrinsic amplitudes due to geometric cancellation. The points that form the upper envelopes in both panels of Figure 2 are then better indicators of the intrinsic amplitude at that temperature. Note that in the few cases of underresolved data, the beating of closely spaced periodicities can lead us to determine a relatively smaller or larger amplitude than the observable amplitude.

We expect that ZZ Ceti stars with a high pulsation amplitude have a corresponding higher uncertainty in temperature. For largeamplitude variables, the surface temperature changes substantially during a pulsation cycle, by as much as $1000 \mathrm{~K}$ (McGraw 1979). Depending on which phase of the pulsation cycle (typical periods of 600-1200 s for cool DAVs) we acquire the spectra for and for how long, our measure of their effective temperature can be incorrect by a few hundred $\mathrm{K}$. We can attempt to estimate this uncertainty by obtaining time-series photometry on the star simultaneous with the spectroscopic data.

At any given temperature, the apparently low-amplitude variables could be suffering from extensive geometric cancellation. At the same time, the high-amplitude variables with the least expected geometric cancellation have a proportionally high uncertainty in temperature due to the large intrinsic temperature fluctuation during a pulsation cycle. This makes the task of interpreting Figure 2 difficult. There is, fortunately, a silver lining to this bleak cloud; ZZ Ceti stars are multimode pulsators. If a ZZ Ceti with three independent modes still exhibits a small amplitude, then it is unlikely that we are dealing with an unfavorable inclination angle in all three cases. ${ }^{5}$

Instead of traditional histograms, we adopt a Gaussian binning technique in which each bin serves as a Gaussian function instead of a box (top hat) function. This is a better noise-averaging technique, because all points contribute to the value of each bin. However, this is not a suitable method of studying sharp local trends because of the finite and nonzero contribution of distant points to the value of an individual bin. We do not hesitate to use this technique because we are interested in slow trends across the width of the instability strip. We choose Gaussian bins with $\sigma=75 \mathrm{~K}$ that are an infinitesimal $1 \mathrm{~K}$ apart from each other. We show the result as a solid line in both panels of Figure 2. Both samples show an initial increase in pulsation amplitude and are also suggestive of a decline prior to the red edge.

We established in $\S 2.2$ how the weighted mean pulsation period correlates directly with the effective temperature of the DAVs for both samples. We now merge both the SDSS and the BG04 samples, also including the five DAVs with unreliable spectroscopic fits (see $\S 1.1)$ to plot the pulsation amplitude as a function of the WMP. We no longer have to worry about the internal consistency in their spectroscopic temperatures, and we can use their WMP as a $T_{\text {eff }}$ scale. We show these 81 DAVs as circles in Figure 3. We also include the 19 new DAVs from Silvotti et al. (2005, 2006), Castanheira et al. (2005), and Kepler et al. (2005) as squares. We have excluded the massive DAV WD $1337+0104(\log g=8.55)$ from Kepler et al. (2005) and the low-mass DAVs HE 0031-5525 ( $\log g=7.65)$ and WD 2135-0743 $(\log g=7.67)$ from Castanheira et al. (2005) in Figure 3.

Figure 3 shows a plot with better statistics than Figure 2 due to the larger sample size of 100 DAVs. We used Gaussian bins of width $75 \mathrm{~s}$, separated from adjacent bins by an infinitesimal amount of $1 \mathrm{~s}$, to produce both the curves shown in Figure 3. The solid line is the histogram determined from the 81 averagemass DAVs of the BG04 and SDSS samples, while the dotted line shows the effect of including the new 19 average-mass DAVs. The minor difference between the two lines assures us that our conclusions are robust. We clearly see an initial increase in pulsation amplitude near the blue edge, followed by a gentle rise and then a decline prior to the red edge.

We find several reasons why Figure 3 looks much more convincing of a decline in amplitude before the red edge compared to Figure 2. First, changing from an $x$-axis based on spectroscopic temperature to one based on weighted mean period allows

\footnotetext{
5 This may not hold true if all the independent modes have the same values of $\ell$ and $m$. However, we do not fully understand the mode selection mechanism for different $m$ values. They also exhibit different cancellation patterns (Dziembowski 1977; Pesnell 1985).
} 


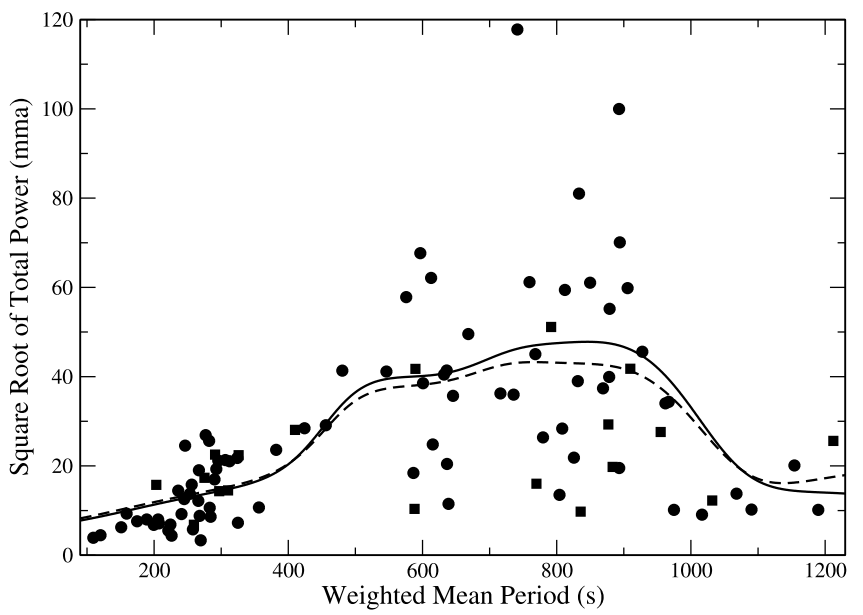

FIG. 3.-Square root of total power for 81 DAVs from the SDSS and BG04 samples (circles) and 19 newly discovered DAVs (squares) as a function of the weighted mean period, which is correlated to the temperature (see Fig. 1). The solid line shows a histogram obtained using Gaussian bins of $\sigma=75 \mathrm{~s}$ for the 81 DAVs, and the dotted line shows the effect of including the 19 new DAVs. We see an initial increase in pulsation amplitude near the blue edge and a subsequent gentle rise followed by a decline in amplitude prior to the red edge of the ZZ Ceti instability strip.

us to include four red-edge pulsators with low amplitudes, namely HS 0952+1816, WD 1443+0134, WD 0249-0100, and WD 2307-0847. Second, we get better statistics by combining the two samples that are individually suggestive of a decline in amplitude near the red edge. Finally, we also find that the change in $x$-axis between Figures 2 and 3 moves pulsators left and right, apparently leaving behind cleaner evidence of a decline prior to the red edge. For example, we find that the low-amplitude cool DAV GD 154 moves from $11,180 \mathrm{~K}$ in Figure 2 to $1154 \mathrm{~s}$ in Figure 3, which corresponds to $T_{\text {eff }} \sim 10,950 \mathrm{~K}$ using the slope in the bottom panel of Figure 1 as a conversion factor.

We have eight pulsators with weighted mean periods of order or greater than $1000 \mathrm{~s}$, with amplitudes near or less than $25 \mathrm{mma}$. There are no high-amplitude pulsators in this range, and the scatter in amplitude near the red edge is much smaller than the center of the ZZ Ceti instability strip. It is unlikely that unfavorable inclination angles can explain the low amplitudes of all the independent modes of all eight pulsators.

Kanaan et al. (2002) established the observed red edge at $11,000 \mathrm{~K}$, but did not see any decline in pulsation amplitude near the red edge within the instability strip, although they were searching for it. It is for the first time that we now have clear evidence of a decline in pulsation amplitude within the instability strip, just prior to the ZZ Ceti red edge. In other words, the star loses pulsation energy before pulsations shut down at the red edge. This work will have implications and new constraints for our pulsation models.

\section{CONCLUSIONS}

We find that the current large sample of noninteracting DAVs conforms to the well-established trend of increasing pulsation periods with decreasing temperature across the instability strip. Investigations of the number of observed independent modes show that hot DAVs have one more mode on average than the cool DAVs. We also confirm the increase in pulsation amplitude near the blue edge and find strong evidence of a decline in amplitude prior to the red edge. Kanaan et al. (2002) established the observed ZZ Ceti red edge at $11,000 \mathrm{~K}$ but did not see any decline in pulsation amplitude near the red edge within the instability strip. We present the first observational evidence that shows the red edge is not an abrupt feature in ZZ Ceti evolution and that the star loses pulsation energy before pulsations shut down at the red edge. This work poses new constraints on our pulsation models.

Support for this work was provided by NASA through the Hubble Fellowship grant HST-HF-01175.01-A awarded by the Space Telescope Science Institute, which is operated by the Association of Universities for Research in Astronomy, Inc., for NASA, under contract NAS 5-26555. We thank the referee for helping us make this manuscript a better paper.

\section{REFERENCES}

Bergeron, P., Fontaine, G., Billères, M., Boudreault, S., \& Green, E. M. 2004, ApJ, 600, 404

Bergeron, P., \& Lamontagne, R. 2003, in White Dwarfs, ed. D. de Martino et al. (Dordrecht: Kluwer), 219

Bergeron, P., Wesemael, F., Lamontagne, R., Fontaine, G., Saffer, R. A., \& Allard, N. F. 1995, ApJ, 449, 258

Bergeron, P., et al. 1993, AJ, 106, 1987

Brassard, P., Fontaine, G., \& Wesemael, F. 1995, ApJS, 96, 545

Brickhill, A. J. 1992, MNRAS, 259, 529

Castanheira, B. G., et al. 2004, A\&A, 413, 623 . 2005, A\&A, submitted (astro-ph/0511804)

Clemens, J. C. 1993, Ph.D. thesis, Univ. of Texas at Austin

Cox, J. P. 1980, Theory of Stellar Pulsation (Princeton: Princeton Univ. Press)

Dolez, N. 1998, Baltic Astron., 7, 153

Dziembowski, W. 1977, Acta Astron., 27, 203

Fleming, T. A., Liebert, J., \& Green, R. F. 1986, ApJ, 308, 176

Fontaine, G., Bergeron, P., Billères, M., \& Charpinet, S. 2003, ApJ, 591, 1184

Fontaine, G., Bergeron, P., Brassard, P., Billères, M., \& Charpinet, S. 2001, ApJ, 557, 792

Fontaine, G., Lacombe, P., McGraw, J. T., Dearborn, D. S. P., \& Gustafson, J. 1982, ApJ, 258, 651

Fontaine, G., \& Wesemael, F. 1984, AJ, 89, 1728

Fontaine, G., Wesemael, F., Bergeron, P., Lacombe, P., Lamontagne, R., \& Saumon, D. 1985, ApJ, 294, 339

Gianninas, A., Bergeron, P., \& Fontaine, G. 2005, ApJ, 631, 1100

Giovannini, O., Kepler, S. O., Kanaan, A., Costa, A. F. M., \& Koester, D. 1998, A\&A, 329, L13

Handler, G., Romero-Colmenero, E., \& Montgomery, M. H. 2002, MNRAS, 335,399

Hansen, C. J., Winget, D. E., \& Kawaler, S. D. 1985, ApJ, 297, 544

Hesser, J. E., Lasker, B. M., \& Neupert, H. E. 1976, ApJ, 209, 853

Kanaan, A., Kepler, S. O., \& Winget, D. E. 2002, A\&A, 389, 896

Kanaan, A., et al. 2005, A\&A, 432, 219

Kepler, S. O. 1984, ApJ, 286, 314

Kepler, S. O., Castanheira, B. G., Saraiva, M. F. O., Nitta, A., Kleinman, S. J., Mullally, F., Winget, D. E., \& Eisenstein, D. J. 2005, A\&A, 442, 629

Kepler, S. O., Nather, R. E., McGraw, J. T., \& Robinson, E. L. 1982, ApJ, 254, 676

Kepler, S. O., et al. 1995, ApJ, 447, 874

Kim, A., Winget, D. E., Montgomery, M. H., \& Kepler, S. O. 2005, preprint (astro-ph/0510104)

Kleinman, S. J. 1995, Ph.D. thesis, Univ. of Texas at Austin

Kleinman, S. J., et al. 1998, ApJ, 495, 424

2004, ApJ, 607, 426

Koester, D., \& Allard, N. F. 2000, Baltic Astron., 9, 119

Koester, D., \& Holberg, J. B. 2001, ASP Conf. Ser. 226, 12th European Workshop on White Dwarfs, ed. J. L. Provencal et al. (San Fransisco: ASP), 299

Koester, D., \& Vauclair, G. 1997, in 10th European Workshop on White Dwarfs, ed. J. Isern, M. Hernanz, \& E. Gracia-Berro (Dordrecht: Kluwer), 429

Kotak, R., van Kerkwijk, M. H., \& Clemens, J. C. 2002, A\&A, 388, 219 
McGraw, J. T. 1979, ApJ, 229, 203

.1980 , in Current Problems in Stellar Pulsation Instabilities (NASA N80-25229 15-90; Greenbelt: GSFC), 501

McGraw, J. T., Fontaine, G., Lacombe, P., Dearborn, D. S. P., Gustafson, J., \& Starrfield, S. G. 1981, ApJ, 250, 349

McGraw, J. T., \& Robinson, E. L. 1975, ApJ, 200, L89 1976, ApJ, 205, L155

Montgomery, M. H. 2005, ApJ, 633, 1142

Mukadam, A. S., Kepler, S. O., Winget, D. E., \& Bergeron, P. 2002, ApJ, 580, 429

Mukadam, A. S., Winget, D. E., von Hippel, T., Montgomery, M. H., Kepler, S. O., \& Costa, A. F. M. 2004a, ApJ, 612, 1052

Mukadam, A. S., et al. 2003, ApJ, 594, 961 2004b, ApJ, 607, 982

Mullally, F., Thompson, S. E., Castanheira, B. G., Winget, D. E., Kepler, S. O., Eisenstein, D. J., Kleinman, S. J., \& Nitta, A. 2005, ApJ, 625, 966

Nather, R. E., \& Mukadam, A. S. 2004, ApJ, 605, 846

O'Donoghue, D. E., \& Warner, B. 1982, MNRAS, 200, 563

O’Donoghue, D., Warner, B., \& Cropper, M. 1992, MNRAS, 258, 415

Pesnell, W. D. 1985, ApJ, 292, 238

Pfeiffer, B., et al. 1995, Baltic Astron., 4, 245

Robinson, E. L. 1979, IAU Colloq. 53, White Dwarfs and Variable Degenerate Stars (New York: Springer), 343

- 1980, in Current Problems in Stellar Pulsation Instabilities (NASA N80-25229 15-90; Greenbelt: GSFC), 423

Robinson, E. L., Kepler, S. O., \& Nather, R. E. 1982, ApJ, 259, 219
Robinson, E. L., \& MacGraw, J. T. 1976, ApJ, 207, L37

Robinson, E. L., et al. 1995, ApJ, 438, 908

Silvotti, R., Pavlov, M., Fontaine, G., Marsh, T., \& Dillon, V. 2006, Mem. Soc. Astron. Italiano, 76(4), in press

Silvotti, R., Voss, B., Bruni, I., Koester, D., Reimers, D., Napiwotzki, R., \& Homeier, D. 2005a, A\&A, 443, 195

Stobie, R. S., Chen, A., O'Donoghue, D., \& Kilkenny, D. 1993, MNRAS, 263, L13

Stobie, R. S., O’Donoghue, D., Ashley, R., Koen, C., Chen, A., \& Kilkenny, D. 1995, MNRAS, 272, L21

Thompson, S. E., Clemens, J. C., van Kerkwijk, M. H., O’Brien, M. S., \& Koester, D. 2004, ApJ, 610, 1001

Unno, W., Osaki, Y., Ando, H., Saio, H., \& Shibahashi, H. 1989, Nonradial Oscillations of Stars (2nd ed.; Tokyo: Univ. of Tokyo Press)

Vauclair, G., Dolez, N., \& Chevreton, M. 1987, A\&A, 175, L13

Vauclair, G., Dolez, N., Fu, J.-N., \& Chevreton, M. 1997, A\&A, 322, 155

Vauclair, G., Dolez, N., Fu, J.-N., Homeier, D., Roques, S., Chevreton, M., \& Koester, D. 2000, A\&A, 355, 291

Vauclair, G., Goupil, M. J., Baglin, A., Auvergne, M., \& Chevreton, M. 1989, A\&A, 215, L17

Weidemann, V. 1990, ARA\&A, 28, 103

Winget, D. E. 1982, Ph.D. thesis, Univ. of Rochester

Winget, D. E., \& Fontaine, G. 1982, in Pulsations in Classical and Cataclysmic Variable Stars, ed. J. P. Cox \& C. J. Hansen (Boulder: JILA), 46

Wu, Y. 2001, MNRAS, 323, 248 